DOI: $10.17805 /$ zpu.2016.4.8

\title{
Социотехнологические предпосылки становления персонализированной медицины
}

\author{
Р. Р. БЕЛЯЛЕТДИНОВ \\ (ИНСТИТУТ ФИЛОСОФИИ РОССИЙСКОЙ АКАДЕМИИ НАУК)
}

В статье рассматриваются особенности становления персонализированной медицины как социотехнологического артефакта. С точки зрения теории исследований науки и технологий ценности общества оказывают фундаментальное влияние на развитие технологий. Научный проект как совокупность работающих технологий превращается в социотехнологический артефакт в том случае, если он признан обществом. Даже нефункционирующие технологии могут рассматриваться как работающие, если они получают общественное признание.

Сторонники персонализированной медицины исходят из того, что люди с некоторыми особенностями, обнаруживаемыми с помощью генетических тестов, лучше реагируют на определенные лекарства. Так, фармакологическая компания Roche имела в своем активе лекарство Xeloda, которое, попав в организм, переходило в активное состояние под воздействием определенных ферментов, встречающихся только у некоторых пациентов.

Персонализированная медицина опирается на самые современные достижения науки и техники, причем не только биомедицины, но и информационных технологий, связанных с хранением, анализом и построением алгоритмов обработки больших объемов информации. В связи с этим развитие персонализированной медицины сопряжено с технологическими трудностями. Несмотря на то что биологические данные позволяют не только делать новые обобщения и устанавливать биологические закономерности с помощью «омиков», они также несут в себе большое число ошибок на уровне хранения и обработки значительных объемов информации. Тем не менее это не мешает рассматривать персонализированную медицину как многообещающий проект, реализующий представления о медицине будущего.

Становление персонализированной медицины предлагается рассмотреть как развитие идеи, согласно которой технодетерминизм выступает в качестве социогуманитарной ценности, а персонализированная медицина представляет собой проекцию социогуманитарного технодетерминизма на конвенциональную медицину.

Ключевые слова: технодетерминизм; персонализированная медицина; STS; принцип симметрии 


\section{ВВЕАЕНИЕ}

\/ нституционально то, как общество оказывает воздействие на развитие техноло- гий, является объектом исследований науки, общества и технологии - Science and Technology Studies (STS). Значимой для STS остается направленность на развенчание мифа о ведущей роли технологий в обществе или технологического детерминизма, в котором технологический прогресс уравнивается с социальным прогрессом (Wyatt, 2008: 168). С позиций STS наука - это не только совокупность технологий, возникших в лаборатории и являющихся самостоятельными артефактами, но и реакция на социальные ценности. Значимость инноваций вытекает не столько из совокупности объективных технических процессов, сколько из готовности общества производить и принимать эти процессы как работающие структуры.

T. Пинч (T. Pinch) и В. Бийкер (W. Bijker) полагают, что если общество признает, что некоторая машина работает, то само это признание превращает машину в успешный артефакт, при этом качество работы этой машины не оказывает влияния на ее успех (Pinch, Bijker, 1984). Точно так же и научный проект как совокупность работающих технологий превращается в социотехнологический артефакт только в том случае, если он признан обществом. Следовательно, даже нефункционирующие технологии могут рассматриваться как работающие, если они получают признание. Можно попробовать рассмотреть персонализированную медицину в этой проекции.

Персонализированная медицина является амбициозной попыткой трансформации конвенциональной медицины (использующей лекарства без учета генетических особенностей пациента). Персонализированная медицина неразрывно связана с техническим прогрессом, поскольку опирается на самые современные достижения науки и техники, причем не только биомедицины, развивающей многочисленные «омики» (геномику, транскриптомику, эпигеномику, протеомику и др.), но и информационных технологий, связанных с хранением, анализом и построением алгоритмов обработки больших объемов информации. Существенная сторона персонализированной медицины как артефакта - это развитие технологий и через них развитие общества (технологический детерминизм). Однако предпосылки развития персонализированной медицины, которые формируют ее социальную базу как артефакта, не столь прямолинейны. Становление персонализированной медицины связано с технологическими трудностями, и реализация проекта вызывает опасения. Между тем общество инвестирует в проект персонализированной медицины свои ожидания, даже невзирая на то, что она пока еще не существует в полной мере как медицинская система, гарантирующая здоровье своим пациентам.

\section{ПЕРСОНААИЗИРОВАННАЯ МЕАИЦИНА: ОТ ПРОСТОГО К САОЖНОМУ}

Идея персонализированной медицины не была напрямую связана с проектом «Геном человека», в котором были предприняты исследования, чтобы определить последовательность нуклеотидов в человеческом геноме и идентифицировать гены, входящие в геном человека. Исследования в области персонализированной медицины не ориентировались на решение подобной масштабной задачи и были направлены на достижение конкретных целей.

В конце 1990-х годов крупные фармакологические компании стали предпринимать попытки применять немногочисленные известные в то время генетические маркеры Аля оптимизации действия уже существующих лекарственных препаратов. Была выдвинута гипотеза о том, что люди с определенными генетическими особенностями 
лучше реагируют на некоторые лекарства. Так, фармакологическая компания Roche имела в своем активе лекарство Xeloda, которое, попав в организм, переходило в активное состояние под воздействием определенных ферментов. Ученые из Roche предположили, что пациенты, не имеющие отклика на лекарство, возможно, обладают персональными нуклеотидными полиморфизмами, отвечающими за генерацию ферментов, которые не переводят лекарство в активное состояние (Langreth, Waldholz, 1999: 426).

Идея проекта по картированию нуклеотидных полиморфизмов, которые отвечают за индивидуальный отклик на лекарственные препараты, была поддержана десятью фармакологическими компаниями, в том числе Roche, Novartis u Glaxo Welcome (там же: 426). Кроме того, первоначальный замысел был расширен до поиска полиморфизмов, ответственных за риски диабета, астмы и болезней сердца.

На сегодняшний день замысел пионеров персонализированной медицины перешел из стадии решения прикладных трудностей отдельных фармакологических компаний в разряд глобальных проектов по смене парадигмы медицины и превращения ее в персональную, превентивную и предиктивную медицину.

\section{БИОИНФОРМАЦИЯ И ПРОБАЕМА ВIG DАТА}

Биоинформация - ключевой аспект развития персонализированной медицины. Она поступает от «омиков» - многочисленных биомедицинских тестов, которые отражают в деталях индивидуальные биологические процессы. Однако лаборатории, генерируя потоки данных, сталкиваются с проблемой их интеграции в единую картину, которую можно было бы полноценно использовать (Alyass, Turcotte, Meyre, 2015). Речь идет о необходимости интеграции тысяч параметров сотен тысяч пациентов. При этом биологические системы - это нелинейные взаимодействия и эффект, возникающий в результате объединения многих факторов, который приводит к сложности идентификации сигнала и ошибки (там же). Чтобы представить себе, как работает персонализированная медицина, следует взглянуть на нее как на процесс превращения данных в клинически полезные результаты.

Хранение биологических данных обычно осуществляется с помощью компьютеризированных суперсистем и решается при помощи облачных сервисов, таких как EasyGenomics (Пекинский институт геномики), информационное облако Embassy, входящее в европейский проект Elixir. Обычные лаборатории не в состоянии поддерживать вычислительные системы, которые могли бы справляться с такими потоками информации.

После поступления биоинформации в облако, ее необходимо обрабатывать. Проблема интеграции данных связана с ограниченностью числа биологических образцов и большим числом тестов, для которых они используются (эта проблема персонализированной медицины известна как «большая размерность» данных, полученных от «омик»-тестов). Поскольку результаты тестов с использованием небольших образцов могут давать отклонения по отношению к данным, полученным от применяемого для исследования биологического материала (как правило, это некоторый конечный набор биологических образцов), совместный анализ данных, полученных из одного источника и интегральных данных, приводит к росту ошибочных результатов (там же).

Биологические данные позволяют не только делать новые обобщения и устанавливать биологические закономерности с помощью «омиков», но также несут в себе большое число ошибок на уровне значительных объемов информации (там же). Так, 
3 гигабайта информации, содержащей расшифровку генома человека, будут иметь 30000 возможных ошибочных данных (Bioinformatics challenges, 2011: 1742).

Решение проблемы интеграции больших объемов информации в единую и содержательную клиническую картину является масштабной технической задачей, которая находится в процессе решения. Тем не менее даже в случае успеха в преодолении проблемы обработки биоинформации перспектива использования «персонализированной» машины может оказаться не столь масштабной. Пока не удается установить, насколько персонализированная медицина может быть эффективна, так как «геном человека не позволяет объяснить вариативность обычных заболеваний, а те особенности, которые он объясняет, как правило, не подпадают под прямые медицинские интервенции» (Joyner, 2015).

\section{ПЕРСОНАИИЗИРОВАННАЯ МЕАИЦИНА \\ И ТЕХНОАОГИЧЕСКИЙ АЕТЕРМИНИЗМ}

Если рассматривать персонализированную медицину как социотехнологический артефакт, т. е. как совместный проект ученых и общества, то необходима методология, которая бы объяснила, каким образом этот проект может развиваться, поглощая значительные объемы научной активности и при этом не решая никаких глобальных проблем общественного здоровья.

Персонализированная медицина появилась в конце 1990-х годов и сегодня она попрежнему остается «обещающей технологией», по сути, технобиомедицинской машиной, работающей без результата, лишь углубляющей собственную проблематику, создающей все новые инновации, завораживающей, но не производящей продукт - повышение качества медицины. На правах гипотезы можно предположить, что существует потребность общества в технологиях, имеющая своей целью не результат их применения, а сам факт поступательного технологического процесса, который может развиваться какой-то период времени только лишь за счет технодетерминистских интенций, производимых самим обществом.

С методологической точки зрения эта проблема может анализироваться с помощью принципа симметрии. Принцип симметрии рассматривается в статье С. Виат (Wyatt, 2008: 165-180), где автор экстраполирует этот метод на отношения аналитиков и акторов (исследователей социогуманитарных процессов в обществе, связанных с технологиями, и участников этих процессов - простых людей, ученых, инвесторов). Первоначальная версия принципа симметрии была введена A. Блуром (D. Bloor) Аля социологии науки. Он полагал, что как истинные, так и ложные утверждения должны рассматриваться с точки зрения социологии в терминах социологии. Аюбые отсылки к природе как аргументу в пользу истинности того или иного утверждения принимать не следует, так как само признание чего-либо природой не является объективным фактом, а конъюнкцией природного и истинного, поэтому любые референции к аргументу от природы необходимо устранять. Перенося этот принцип на отношение аналитиков и акторов, С. Виат полагает, что «то, каким образом стейкхолдеры (заинтересованные лица. - P. Б.) и аналитики идентифицируют других игроков и их интересы, следует толковать симметрично» (Wyatt, 2008: 171). Таким образом, автор развивает идею Э. Гидденса о том, что социальные ученые должны не только генерировать теории, объясняющие действия социальных акторов, но также им следует принимать во внимание то, как социальные игроки воспринимают их теории. И поскольку технодетерминизм в различных формах присутствует в социальном пространстве 
(там же), технодетерминистский дискурс необходимо включать в анализ происходящего в обществе.

Особенно интересно отметить, что выделение трех типов медицины: «у постели больного», «больничная медицина» и «лабораторная медицина» (Tutton, 2012: 6) — отражает изменение статуса пациента и его выпадение из процесса лечения, но не касается того, что и врач выпадает из процесса лечения, предоставляя синтез «омиков» сложным вычислительным машинам. В этом смысле под видом персонализации можно увидеть стремление общества к глобальному социотехнологическому артефакту «машине», реализованной в виде обезличивающей супергенерализированной медицины, из которой уже исключены и врач, и пациент, - остается только вычислительная система, геном и оцифрованные модели заболеваний.

\section{ЗАКАЮЧЕНИЕ}

Персонализированная медицина представляет собой проекцию социального «естественного» технодетерминизма на конвенциональную медицину. Если следовать идее, что «работающая» машина является результатом признания, а не причиной, то «ожидание» персонализированной медицины есть признание ценности артефакта вне зависимости от его фактической полезности. И пусть критики персонализированной медицины запрашивают новые модели обоснования, которые бы поддерживали ее развитие. В этом нет необходимости, поскольку в обществе есть позитивное отношение к технологиям как инструментам достижения результата.

В настоящее время персонализированная медицина проявляет себя не столько в результатах, сколько в технологиях обработки и интерпретации биоинформации и развитии компьютерных систем работы с биоинформацией. Как социотехнологический артефакт, персонализированная медицина развивается в значительной степени за счет «социологии ожидания», одновременно внося весомый вклад в развитие компьютерных технологий. Но очень симптоматично то обстоятельство, что для конвенциональной медицины как сложившейся традиции персонализированная медицина в своем несовершенном виде выглядит чужеродным методом лечения, закрытым для большинства врачей в силу технологических особенностей обработки информации «омиков».

\section{СПИСОК АИТЕРАТУРЫ}

Alyass, A., Turcotte, M., Meyre, D. (2015) From big data analysis to personalized medicine for all: challenges and opportunities // BMC Medical Genomics. V. 8. № 33. DOI: 10.1186/s12920-015-0108-y

Bioinformatics challenges for personalized medicine (2011) / G. H. Fernald, E. Capriotti, R. Daneshjou, K. J. Karczewski, R. B. Altman // Bioinformatics review. V. 27. № 13. P. 1741-1748. DOI: 10.1093/bioinformatics/btr295

Fernald, G. H., Capriotti, E., Daneshjou, R., Karczewski, K. J., Altman, R. B. (2011) Bioinformatics challenges for personalized medicine // Bioinformatics review. V. 27, №13. P. 1741-1748. DOI: $10.1093 /$ bioinformatics/btr295

Joyner, M. J. (2015) Seven Questions for Personalized Medicine // 09/2015, V. 314, No. 10. DOI: 10.1001/jama.2015.7725

Langreth, R. and Waldholz, M. (1999) New Era of Personalized Medicine. Targeting Drugs For Each Unique Genetic Profile // The Oncologist, 1999, № 4, P. 426-427.

Pinch, T. J., Bijker, W. E. (1984) The Social Construction of Facts and Artefacts: or How the Sociology of Science and the Sociology of Technology might Benefit Each Other // Social Studies of Science. № 14. P. 399-441. DOI: 10.1177/030631284014003004 
Tutton, R. (2012) Personalizing medicine: Futures present and past // Social Science \& Medicine.

Wyatt, S. (2008) Technological Determinism Is Dead; Long Live Technological Determinism // The Handbook of Science and Technology Studies Third Edition, ed. by Hackett E. J., Amsterdamska O., Lynch M., Wajcman J. The MIT Press, Cambridge, USA. 2008. P. 165-180.

Аата поступления: 05.09.2016 2.

SOCIO-TECHNOLOGICAL BACKGROUND OF THE RISE OF PERSONALIZED MEDICINE

R. R. BELYALETDINOV

(INSTITUTE OF PHILOSOPHY, RUSSIAN ACADEMY OF SCIENCES)

The article discusses the features of the rise of personalized medicine as a socio-technological artifact. From the point of view of the theory of science and technology studies, the values of a society have a fundamental influence on its technological development. A research project as a collection of working technologies can become a socio-technological artifact only if it is recognized by society. Even non-functioning technology can be considered operational if they receive public recognition.

Proponents of personalized medicine proceed from the point that people with certain features detected by genetic testing respond better to certain medications. Thus, pharmaceutical company Roche developed the drug Xeloda, which, once administered, becomes active under the influence of specific ferments found only in some patients.

Personalized medicine is based on the latest achievements of science and technologies, not only in biomedicine, but also in the technologies of storing, analyzing and processing large volumes of information. In this regard, the development of personalized medicine involves certain technical challenges. Although biological big data help arrive at new generalizations an discover biological patterns via OMIC, they also display a large number of errors in storing and processing large volumes of information. Nevertheless, this should not deter us from considering personalized medicine a promising project which implements some ideas associated with the future of medicine.

We suggest that the rise of personalized medicine should be seen in the light of technological determinism becoming a humanist value. In this way, personalized medicine represents a projection of the socio-humanitarian technological determinism upon conventional medicine.

Keywords: technological determinism; personalized medicine; STS; principle of symmetry

Белялетдинов Роман Рифатович - младший научный сотрудник сектора гуманитарных экспертиз и биоэтики Института философии Российской академии наук. Адрес: 109240, Россия, г. Москва, ул. Гончарная, А. 12, стр. 1. Тел.: + 7 (495) 697-90-67. E-mail: roman_rb@iph.ras.ru

Belyaletdinov Roman Rifatovich, Junior Research Fellow, Department of Humanitarian Expertise and Bioethics, Institute of Philosophy, Russian Academy of Sciences. Address: 12 Bldg.1, Goncharnaya St., Moscow, Russian Federation 109240. Tel .: + 7 (495) 697-90-67. E-mail: roman_rb@ iph.ras.ru 